\title{
Physical Entrapment
}

National Cancer Institute

\section{Source}

National Cancer Institute. Physical Entrapment. NCI Thesaurus. Code C50548.

Patient becomes entangled or caught in a bed, particularly in side rails, or mattress, or head/foot boards. 